\title{
Characterization of S-layer proteins of Lactobacillus by FTIR spectroscopy and differential scanning calorimetry
}

\author{
P. Mobili ${ }^{\mathrm{a}, \mathrm{b}}$, A. Londero $^{\mathrm{a}}$, T.M.R. Maria ${ }^{\mathrm{b}}$, M.E.S. Eusébio ${ }^{\mathrm{b}}$, G.L. De Antoni ${ }^{\mathrm{a}}$, R. Fausto ${ }^{\mathrm{b}}$, \\ A. Gómez-Zavaglia ${ }^{\mathrm{b}, \mathrm{c}, *}$ \\ ${ }^{a}$ Centro de Investigación y Desarrollo en Criotecnología de Alimentos, La Plata RA-1900, Argentina \\ ${ }^{\mathrm{b}}$ Department of Chemistry, University of Coimbra, Coimbra P-3004-535, Portugal \\ ${ }^{\text {c } F a c u l t a d ~ d e ~ F a r m a c i a ~ y ~ B i o q u i ́ m i c a, ~ U n i v e r s i d a d ~ d e ~ B u e n o s ~ A i r e s, ~ B u e n o s ~ A i r e s ~ R A-1113, ~ A r g e n t i n a ~}$
}

\section{A R T I C L E I N F O}

\section{Article history:}

Received 1 February 2008

Accepted 28 July 2008

Available online 19 August 2008

\section{Keywords:}

S-layer

Lactobacillus kefir

Lactobacillus brevis

FTIR, DSC

Secondary structure

Proteins

\begin{abstract}
A B S T R A C T
FTIR spectroscopy was used for the characterization of S-layer proteins extracted from microorganisms isolated from kefir grains. S-layer from Lactobacillus brevis ATCC 8287 has been already characterized [G. Vidgren, I. Palva, R. Pakkanen, K. Lounatmaa, A. Palva, J. Bacteriol. 174 (1992) 7419] and therefore it was used for the validation of FTIR as a method to investigate the secondary structure of the S-layer proteins of the studied kefir strains. A correlation between the secondary structures of S-layer proteins with surface properties of Lactobacillus kefir strains was found: a high percentage of $\beta$-sheet contents $(40-50 \%)$ was found for non-aggregating strains, whereas this percentage decreased to $25-30 \%$ for aggregating ones. A quantitative comparison of the S-layers was performed by means of cluster analysis based on the obtained spectroscopic data. This analysis enabled the strains to be grouped in clusters according to the spectral diversity in the Amide I region. The non-aggregating strains of $L$. kefir cluster at $S_{\mathrm{sm}}>0.943$ and the aggregating strains form another cluster, with $S_{\mathrm{sm}}>0.769$. L. brevis ATCC 8287 appears clearly separated from these two clusters: the similarity with the aggregating strains is 0.658 and the similarity with the non-aggregating ones, 0.665 . The thermal analysis of the lyophilized S-layer proteins was performed by means of differential scanning calorimetry (DSC) and FTIR. DSC analysis within the 30$130{ }^{\circ} \mathrm{C}$ range showed two phase transitions with maxima located at $c a .58$ and $98{ }^{\circ} \mathrm{C}$ for $\mathrm{L}$. brevis and in the 67-70 and $110-119{ }^{\circ} \mathrm{C}$ ranges for the different strains of $L$. kefir (CIDCA 8344 only shows the lowest temperature phase transition). FTIR spectra obtained reveal that for all the $L$. kefir S-layer proteins the major secondary structure modifications upon heating occur nearly at the first phase transitions observed by DSC, with the thermal stability increasing with the percentage of $\beta$-sheets structures. The Slayer protein of $L$. brevis ATICC 8287, which among all protein studied is that with maximum $\beta$-sheet contents (and no $\alpha$-helix structure) was then found to be the protein showing a greater thermal stability.

(c) 2008 Elsevier B.V. All rights reserved.
\end{abstract}

\section{Introduction}

Kefir is a fermented milk that has been consumed for thousands of years. It was originated from the northern area of the Caucasus Mountains and it is prepared from kefir grains [2]. Kefir grains are clusters of microorganisms that include primarily lactic acid bacteria (lactobacilli, lactococci, leuconostoc), yeasts, acetic acid bacteria and other microorganisms not yet described held together by a matrix of polysaccharides and proteins [3-5]. These

\footnotetext{
* Corresponding author at: Department of Chemistry, University of Coimbra, Coimbra P-3004-535, Portugal.

E-mail address: angoza@qui.uc.pt (A. Gómez-Zavaglia).
}

microorganisms are usually associated with the beneficial effects of kefir. This beneficial activity is at least partially related with bacterial surface properties, as adhesion and aggregation, which, in turn, depend on the specific characteristics of their S-layer. ${ }^{1}$

The S-layer is a crystalline bi-dimensional array of glycoproteic subunits, linked non-covalently to each other and to the underlying cell wall $[6,7]$. S-layer subunits are generally assembled in lattices with oblique, square or hexagonal symmetry and show the intrinsic tendency to reassemble into two-dimensional arrays after removal of the disrupting agent used in the extraction procedure [6]. Several specific functions have been reported for S-layers, such

\footnotetext{
${ }^{1}$ The S-layer ("surface layer") is the most external layer in bacteria.
} 
as protective coats, molecular sieves, molecule and ion traps, structures involved in cell adhesion and surface recognition, and virulence factors. The presence of S-layer proteins in Lactobacillus kefir and Lactobacillus parakefir isolated from kefir grains has been reported for the first time in the last years [8].

Taking into account that several Lactobacillus species bearing an S-layer structure play an essential role in many fermentation processes in food industry, silage fermentations and in probiotics for humans and animals, elucidation of the characteristics of lactobacilli S-layers has become increasingly important [9].

FTIR is a well-established methodology that has been extensively used for the elucidation and analysis of the secondary structure of proteins [1,10-12]. The singular advantage of FTIR over other techniques is that spectra can be obtained for proteins in a wide range of environments, e.g., in the crystalline state, solution and on different surfaces, including polymers, metals and bioceramics [13,14 among many other authors].

There is a wealth of information that can be used to obtain structural information by analyzing the shape and position of bands in the Amide I region of infrared spectra $\left(1720-1600 \mathrm{~cm}^{-1}\right)$. Indeed, protein secondary structural elements, like the $\alpha$-helix, $\beta$ sheets and random coil structures, have characteristic absorbance bands in the Amide I region [15-18]. Because these bands are considerably broad and overlap significantly, the use of deconvolution or second-derivative techniques is required to mathematically enhance the resolution of the spectrum, separating bands that are intrinsically broader than the instrument resolution [1921]. These are, however, well-established procedures since long ago. Empirical association was made between the position of Amide I band and/or the position and relative areas of component bands after deconvolution and the type and relative content of secondary structure in the protein $[22,10,23]$.

The information obtained by infrared spectroscopy can also be enriched by complementary analysis by other methods. Differential scanning calorimetry (DSC) is a very powerful method for the study of phase transition processes, providing very precise and direct measure of the associated heat fluxes. It has been used to investigate the melting temperatures of proteins as well as protein denaturation and the results successfully correlated with the changes observed in the Amide I infrared spectral region (and also, less frequently, in other spectral regions) upon heating [12,24].

FTIR has been used for the characterization of S-layers from a few microorganisms, such as Acetogenium kivui [25] and Bacillus sphaericus [26]. However, to the best of our knowledge it has not been used for the analysis of S-layers of lactobacilli hitherto. Therefore, the aim of this work was to investigate the secondary structure of S-layer proteins extracted from several $L$. kefir and
Lactobacillus brevis strains, which have been shown to have different surface properties. The relationship between the secondary structure of the S-layer proteins and the surface properties of the corresponding whole microorganisms was then analyzed. Finally, the behavior of the S-layer proteins upon heating was also studied by combining DSC and FTIR methods.

\section{Materials and methods}

\subsection{Bacterial strains and growth conditions}

Reference strains of $L$. brevis ATCC 8287 and L. kefir JCM 5818 and four strains of $L$. kefir isolated from kefir at CIDCA (L. kefir CIDCA 83113, CIDCA 8321, CIDCA 8344 and CIDCA 8348) were cultured in MRS broth [27] (Biokar Diagnostics, Beauvais, France) at $30{ }^{\circ} \mathrm{C}$ for $48 \mathrm{~h}$. These bacterial strains showed differences in the surface properties (i.e. aggregation and co-aggregation ability), in the S-layer protein molecular mass [8], glycosylation and pattern of recognition by antibodies [28] (Table 1 ).

\subsection{S-layer protein preparation}

Bacterial cells were harvested at stationary phase, collected by centrifugation $\left(10,000 \times \mathrm{g}\right.$ at $10^{\circ} \mathrm{C}$ for $\left.10 \mathrm{~min}\right)$, washed twice with phosphate buffered saline (PBS, $\mathrm{pH} 7$ ), and resuspended to an $\mathrm{OD}_{550 \mathrm{~nm}}$ of 10 in the same buffer. Cells were mixed with $5 \mathrm{M} \mathrm{LiCl}$ (J.T. Baker, Mallinckrodt Baker S.A., Mexico) in a proportion of $4 \mathrm{~mL}$ of solution per $\mathrm{mL}$ of bacterial suspension. The mixture was incubated in a shaking incubator (Environ Shaker, Lab-line Instruments Inc., Melrose Park, IL, USA) at $200 \mathrm{rpm}$ and $37^{\circ} \mathrm{C}$ for $60 \mathrm{~min}$ to extract non-covalently bound proteins. Then, the mixture was centrifuged $\left(12,000 \times \mathrm{g}\right.$ at $10^{\circ} \mathrm{C}$ for $\left.15 \mathrm{~min}\right)$. The supernatant containing the S-layer protein was concentrated by ultrafiltration in an Amicon stirred cell 8050 (Millipore Corporation, US) equipped with a regenerated cellulose membrane (Ultracell PLGC04310, MWCO 10000, Millipore Corporation, US), and dialyzed against bi-distilled water at $4{ }^{\circ} \mathrm{C}$ for $24 \mathrm{~h}$ using a cellulose membrane (SpectraPor membrane tube, MWCO 60008000, Spectrum Medical Industries, California, US). The dialyzed extract was lyophilized in a FD4 Heto freeze drier (Lab Equipment, Denmark) and conserved at room temperature.

\subsection{FTIR spectroscopy}

FTIR spectra of the solid proteins in $\mathrm{KBr}$ pellets (ratio $1 \mathrm{mg}$ protein/100 $\mathrm{mg} \mathrm{KBr}$ ), in the $4000-500 \mathrm{~cm}^{-1}$ range, were recorded in transmission mode in a system continuously purged under dry

Table 1

Surface properties of bacteria used in this study

\begin{tabular}{|c|c|c|c|c|c|c|}
\hline \multirow[t]{2}{*}{ Bacterial strain } & \multirow[t]{2}{*}{$\mathrm{MW}^{\mathrm{a}}(\mathrm{kDa})$} & \multicolumn{2}{|c|}{ S-layer protein reactivity with Abs antisera ${ }^{\mathrm{b}}$} & \multicolumn{3}{|c|}{ Aggregates with } \\
\hline & & $\mathrm{a} 21$ & a87 & Auto $^{c}$ & Yeast $^{\mathrm{d}}$ & Salmonella ${ }^{\mathrm{e}}$ \\
\hline L. kefir CIDCA 8321 & 66 & + & - & + & + & + \\
\hline L. kefir CIDCA 8348 & 66 & + & - & + & + & + \\
\hline L. kefir JCM 5818 & 69 & + & - & - & - & nd \\
\hline L. kefir CIDCA 83113 & 66 & + & - & - & - & - \\
\hline L. kefir CIDCA 8344 & 71 & + & - & - & - & - \\
\hline L. brevis ATCC 8287 & 46 & - & + & - & nd & nd \\
\hline \multicolumn{7}{|c|}{$\begin{array}{l}\text { nd not determined. } \\
\text { a Molecular mass of the S-layer proteins [1,8]. } \\
\text { b Reactivity with rabbit antisera anti-S-layer protein from L. kefir CIDCA } 8321 \text { (a21) or L. brevis ATCC 8287 (a87) [28]. } \\
\text { c Ability to autoaggregate as determined by optical density decay of pure or mixed cell suspensions in phosphate buffered saline (PBS) [38]. } \\
\text { d Ability to coaggregate with yeast Saccharomyces lipolytica CIDCA } 812 \text { as determined by optical density decay of pure or mixed cell suspensions in phosphate buffered } \\
\text { saline (PBS) [38]. } \\
\text { e Ability to coaggregate with Salmonella enterica serovar Enteritidis, as determined by optical density decay of pure or mixed cell suspensions in phosphate buffered saline } \\
\text { (PBS) [38]. }\end{array}$} \\
\hline
\end{tabular}


nitrogen in order to eliminate spectral contributions of atmospheric water vapor and $\mathrm{CO}_{2}$. The IR spectra collected at room temperature were obtained co-adding 512 scans with $4 \mathrm{~cm}^{-1}$ spectral resolution. Temperature-dependent spectra were obtained using a Specac variable temperature infrared cell controlled by a Red Lions digital temperature controller; samples were heated in 5 or $10^{\circ} \mathrm{C}$-intervals and stabilized for $5 \mathrm{~min}$ before a total of 64 scans were co-added (spectral resolution: $4 \mathrm{~cm}^{-1}$ ).

All spectra were recorded on a BOMEM MB40 spectrometer with a $\mathrm{Zn} / \mathrm{Se}$ beam splitter and a DTGS detector.

\subsection{Data analysis of FTIR spectra}

The recorded infrared spectra were analyzed using the OMNIC suit of programs (Nicolet Instrument Co., Madison, WI, USA). Whenever necessary, residual contributions due to atmospheric water vapor and $\mathrm{CO}_{2}$ were eliminated by subtraction of the corresponding spectra from the registered samples' spectra, in order to obtain a flat baseline between 2300 and $1850 \mathrm{~cm}^{-1}$ [29]. The resultant protein spectra were smoothed with a seven-point $\left(13 \mathrm{~cm}^{-1}\right)$ Savitzky-Golay function.

Inverted second derivative spectra were used to estimate the number, position and relative contribution of individual elements composing Amide I band (1600-1720 $\left.\mathrm{cm}^{-1}\right)$, and this information was taken into account to fit Amide I bands in protein spectra with Gaussian band profiles, using OriginPro 7.5 (OriginLab Corporation, USA).

The assignment of protein secondary structures to the principal Amide I frequencies was as previously described in literature $[22,10,23,30]$.

\subsection{Cluster analysis}

The Amide I frequencies (to within $1 \mathrm{~cm}^{-1}$ ) obtained in the second derivative analysis were converted into a double entrance table where the presence or absence of each peak was assigned for each strain as 1 and 0 , respectively. The simple-matching coefficient $\left(S_{\mathrm{sm}}\right)$ was calculated as

$S_{\mathrm{sm}}=\frac{a+c}{a+b+c}$

where $a$ and $c$ correspond to the number of matching present and absent peaks, respectively, and $b$ represents the number of nonmatching peaks between pairs of strains. The $S_{\mathrm{sm}}$ between any pair of strains was computed. The matrix of the $S_{\text {sm }}$ correlation coefficients was clustered by the unweighted average linkage method [31-34] using SYSTAT (Version 12.0).

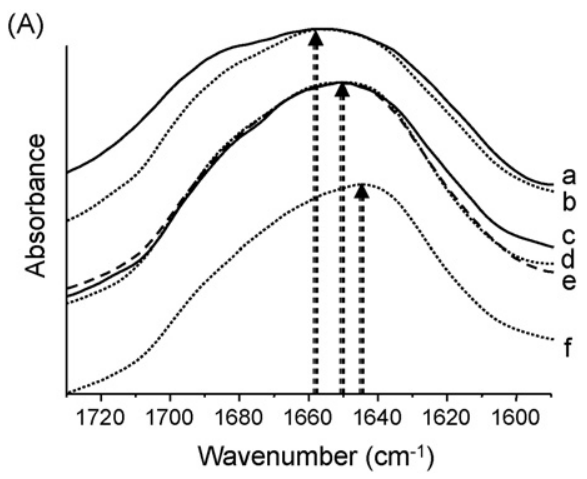

\subsubsection{DSC analysis}

A PerkinElmer DSC7 calorimeter was used with an intracooler unit (with circulating ethyleneglycol:water $1: 1$ mixture at $-15^{\circ} \mathrm{C}$ ). The calorimeter was calibrated for temperature [35] with biphenyl $T_{\text {fus }}: 68.93^{\circ} \mathrm{C}$ and $99.99 \%$ indium $\left(T_{\text {fus }}=156.60{ }^{\circ} \mathrm{C}\right)$. The calibration was confirmed with naphthalene $\left(T_{\text {fus }}=80.20 \pm 0.05^{\circ} \mathrm{C}\right)$ and benzophenone (Metler Toledo Calibration substance, $T_{\text {fus: }}$ : $\left.48.1 \pm 0.2^{\circ} \mathrm{C}\right)$. A $20 \mathrm{~mL} / \mathrm{min}$ nitrogen purging gas flow was used. Samples of lyophilized S-layer proteins weighing ca. 2-4 mg were encapsulated in hermetic $10 \mu \mathrm{L}$ aluminum pans and accurately weighted. An empty aluminum pan was used as reference. Typically, samples were subjected to heating and cooling cycles between 15 and $130^{\circ} \mathrm{C}$, with a heating rate of $25^{\circ} \mathrm{C} / \mathrm{min}$ and $10^{\circ} \mathrm{C} / \mathrm{min}$ cooling rate.

All experiments were carried out at least three times with samples obtained from different S-layer extractions. The relative differences were reproducible independently of the S-layer extracts used (uncertainty $\leq 1^{\circ} \mathrm{C}$ ).

\section{Results and discussion}

3.1. Characterization of secondary structure of S-layer proteins and its relevance in determining surface properties of the lactobacilli strains

According to previous works carried out in our group, L. kefir strains included in this study have different surface properties (Table 1). Among these properties, aggregation ability is perhaps one of the most important differential characteristics among strains because of its putative role in some activities, such as adhesion to intestinal cells, biofilm formation and pathogen inhibition [36-38]. S-layer proteins were associated with aggregation of $L$. kefir strains, since procedures tending to disassemble the S-layer (such as $5 \mathrm{M} \mathrm{LiCl}$ treatment) abolish their aggregative capacity [8].

Fig. $1 \mathrm{~A}$ depicts the unresolved spectra of the studied S-layer proteins in the Amide I region. At a first glance, it is easy to divide the strains in two groups, the first one giving rise to an Amide I band with absolute maximum centered at $c a .1656-1658 \mathrm{~cm}^{-1}$ and the second one with this maximum centered at $1650 \mathrm{~cm}^{-1}$. It is interesting to observe that non-aggregating $L$. kefir strains isolated from kefir belong to the first group whereas the aggregating strains belong to the second one. The Amide I band of $L$. brevis ATCC 8287 does not belong to any of these groups and is centered at $1646 \mathrm{~cm}^{-1}$. This analysis induced us to correlate the aggregative character of each strain to a specific structure of the $S$ layer.

The primary components of the Amide I band are mainly generated by $\mathrm{C}=\mathrm{O}$ stretching vibrations of peptide groups, thus

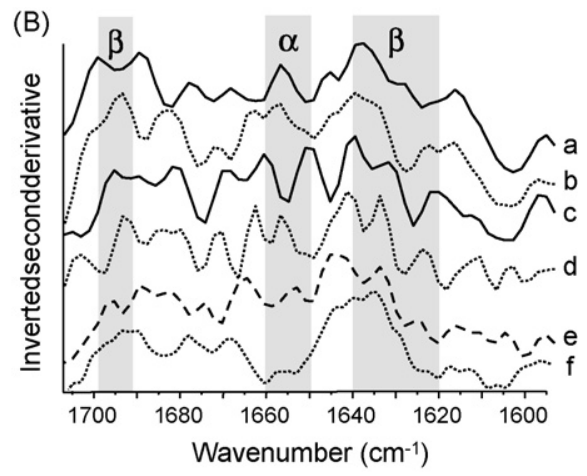

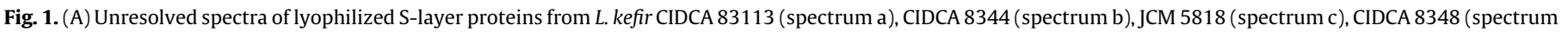

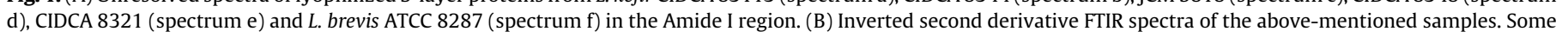
secondary structure assignments in the inverted second derivative spectra are shadowed. 
Table 2

Empirical assignment of secondary structure elements to different amide I band components in proteins $[23,24]$

\begin{tabular}{ll}
\hline Wavenumber $\left(\mathrm{cm}^{-1}\right)$ & Assigned secondary structure \\
\hline $1620-1630$ & Intermolecular $\beta$-sheet \\
$1620-1640$ & Intramolecular $\beta$-sheet \\
$1640-1650$ & No order \\
$1650-1660$ & $\alpha$-Helix \\
$1660-1695$ & $\beta$-Turn \\
1675 & Intramolecular $\beta$-sheet \\
$1690-1700$ & Intermolecular $\beta$-sheet \\
\hline
\end{tabular}

this band is strongly influenced by hydrogen bonds giving rise to elements of secondary structure. Usually, proteins contain different types of secondary structure such as $\alpha$-helices, $\beta$-sheets, turns and non-ordered regions, therefore the Amide I band contour consisting of many overlapping individual component bands at the frequencies that are characteristic of specific secondary structural elements (Table 2). The second derivative analysis permits the direct separation of the Amide I band into its components and thus, provides additional structural information. Absorbance bands in

\section{Cluster Tree}

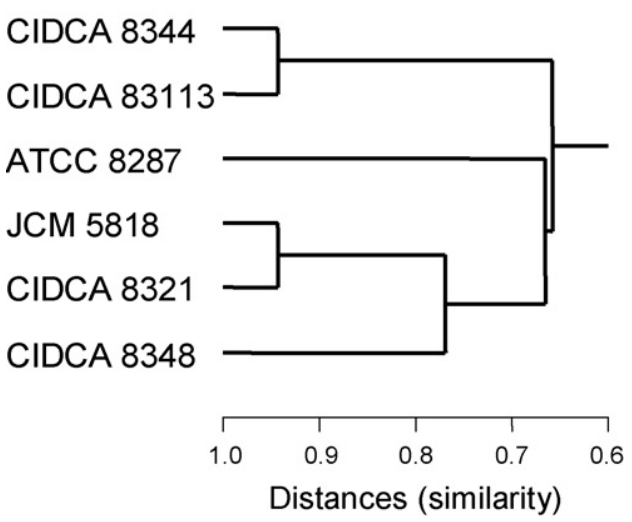

Fig. 2. Dendrogram obtained after unweighted average linkage method analysis of the simple matching coefficient obtained from the frequencies corresponding to the second derivatives FTIR spectra of S-layers from L. kefir and L. brevis ATCC 8287. Similarities are expressed as distances: 1.0 represents $100 \%$ similarity and 0.0 represents $0 \%$ similarity.
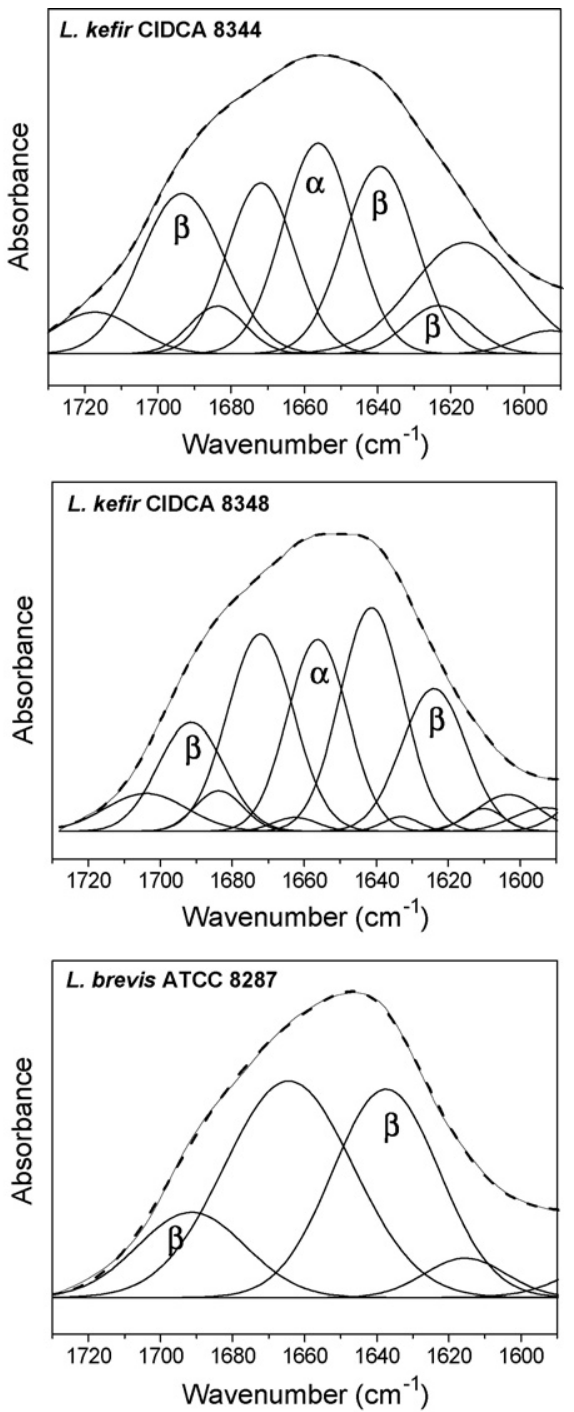

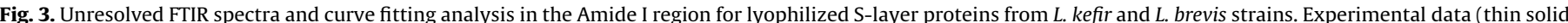

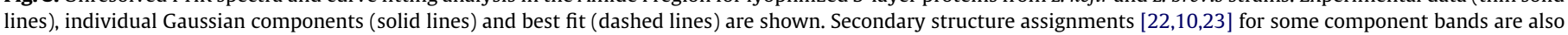
given. 
the original spectrum are revealed as negative bands in the second derivative spectrum, whereby the band position is preserved $[11,12]$. In this context, the analysis of the second derivative of the Amide I bands has been performed for all the strains under study (Fig. 1B). This analysis also points out the existence of two groups of $L$. kefir strains, giving rise to similar second derivative spectra and corresponding to the non-aggregating and aggregating L. kefir strains; as it could be anticipated, $L$. brevis second derivative spectrum profile is substantially different from the ones of the $L$. kefir strains.

These differences were evaluated quantitatively by means of cluster analysis. Such analysis allows a quantitative comparison among strains, because it compares the presence or absence of a given peak for all the strains analyzed. A value within the range 1-0 $\left(S_{\mathrm{sm}}\right)$ must result from this analysis, with $S_{\mathrm{sm}}=1$ corresponding to identity and $S_{\mathrm{sm}}=0$ to absolute non-similarity of the tested samples.

Fig. 2 shows the computerized numerical analysis of the second derivatives of the S-layers, which enabled strains to be grouped in clusters according to the spectral diversity in the Amide I region. The figure shows that the non-aggregating strains of $L$. kefir cluster at $S_{\mathrm{sm}}>0.943$ and the aggregating strains form another cluster, with $S_{\mathrm{sm}}>0.769$. L. brevis ATCC 8287 appears clearly separated from these two clusters: the similarity with the aggregating $L$. kefir strains is 0.658 , whereas the similarity with the non-aggregating $L$. kefir strains is slightly larger 0.665 . The employ of dendrograms has been extensively used for taxonomical purposes [32-34,39-43] but up to our knowledge, it has not been used so far to quantitatively estimate the degree of similarity of structural (or functional) components of biological samples based on spectroscopic information [41-43]. We believe that cluster analysis can indeed play a useful role in the application of FTIR spectroscopy to proteins, as a complement to other, currently used data analysis methods.

Taking into account the second derivative spectra bands as a guide for the location of individual components, Amide I peak fitting using Gaussian functions was performed (Fig. 3). This treatment allowed the estimation of the percentage of different secondary structure components for each lyophilized S-layer protein (Table 3).

The first S-layer protein to be analyzed was the one of $L$. brevis ATCC 8287, because this protein has been already characterized by Vidgren et al. [1]. Its aminoacids composition was deduced from the DNA sequence of the corresponding gene, and consists of a signal peptide of 30 amino acids followed by a protein of 435 aminoacids. The predicted secondary structure for the full polypeptide is composed of $56.5 \% \beta$-sheet and $27.7 \% \alpha$-helix [1].

In this work, the reported aminoacids sequence of the $46 \mathrm{kDa}$ mature protein (without the signal peptide) was used to perform the secondary structure prediction analysis. The methods of Rost and Sander (http://www.predictprotein.org) [44] and Brendel et al. [45] predicted an estimated $\beta$-sheet content of 55.6 and $44.4 \%$, respectively, and no $\alpha$-helix. The contents of $\beta$-sheet structure are higher than those of other S-layer proteins, like the ones extracted from Lactobacillus acidophilus, Lactobacillus crispatus and Lactobacillus helveticus, which have been described as ranging from 20 to $40 \%[25,7,46-48]$, as deduced from circular-dichroism measurements or secondary-structure prediction algorithms. The lack of $\alpha-$ helix structure in the mature protein is in agreement with ÅvallJääskeläinen and Palva [48], which described the $\alpha$-helices as located at the signal sequences of these proteins.

The experimental FTIR spectrum of the S-layer of $L$. brevis ATCC 8287 obtained in this work depicted an Amide I band centered at $1646 \mathrm{~cm}^{-1}$, typical of proteins with high $\beta$-sheet content [23] (Table 2). Peak-fitting for this strain showed four component
Table 3

Amide I position and secondary structure composition (\%) for lyophilized S-layer proteins from lactobacilli, according to relative areas of component bands in peak fitting of amide I zone in original IR spectra

\begin{tabular}{lllll}
\hline Bacterial strain & $\begin{array}{l}\text { Amide I maximum } \\
\text { position }\left(\mathrm{cm}^{-1}\right)\end{array}$ & \multicolumn{3}{l}{ S-layer secondary structure (\%) } \\
\cline { 3 - 5 } & & $\beta$-Sheet & $\alpha$-Helix & Other $^{\mathrm{a}}$ \\
\hline L. kefir CIDCA 8321 & 1651 & 23.0 & 13.6 & 63.4 \\
L. kefir CIDCA 8348 & 1650 & 28.0 & 19.1 & 52.9 \\
L. kefir JCM 5818 & 1651 & 36.0 & 15.3 & 48.7 \\
L. kefir CIDCA 83113 & 1658 & 41.2 & 16.7 & 42.1 \\
L. kefir CIDCA 8344 & 1656 & 42.1 & 21.0 & 36.9 \\
L. brevis ATCC 8287 & 1646 & 50.2 & 0.0 & 49.8 \\
\hline
\end{tabular}

${ }^{a}$ Includes $\beta$-turn and non-ordered structures, as well as side chain vibrations.
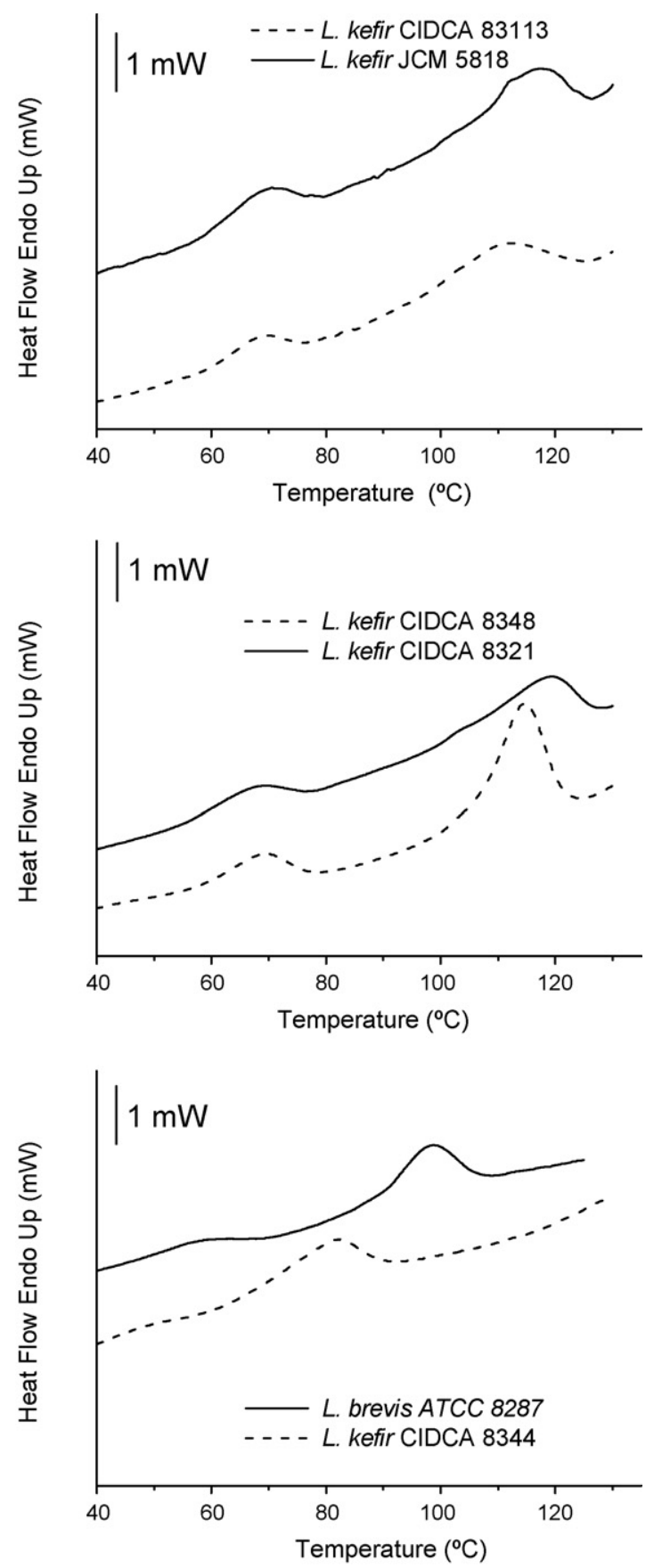

Fig. 4. DSC thermograms for lyophilized S-layer proteins from L. brevis and L. kefir strains. See Section 2 for details. 
bands, two of these with maxima at $\beta$-sheet characteristic frequencies (1637 and $1694 \mathrm{~cm}^{-1}$ ), without any band with maximum in the $1650-1660 \mathrm{~cm}^{-1}$ range ( $\alpha$-helix). The bands corresponding to $\beta$-sheet structures represent $50.2 \%$ of the whole Amide I band (see Fig. 3). This percentage fits nicely the results reported by Vidgren et al. (56.5\%) [1] and those predicted by the methods of Rost and Sander (55.6\%) [44] and Brendel et al. (44.4\%) [45]. This fact can also be considered as a good indication of the reliability of the used approach to estimate the secondary structure motifs of S-layer proteins.

The same procedure was then used to characterize the secondary structure of the lyophilized S-layer proteins from $L$. kefir strains, whose Amide I bands, centered in the 1658$1650 \mathrm{~cm}^{-1}$ range, suggesting the presence of $\alpha$-helix structures in different percentages (Table 2) [10]. As shown in Table 3, the investigated $L$. kefir S-layers showed $\beta$-sheet contents ranging from 23 to $42 \%$ and $\alpha$-helix contents ranging from 13 to $21 \%$. The differences observed in the secondary structures, especially regarding the $\beta$-sheet contents, could be associated with the different aggregative capacities of the $L$. kefir strains analyzed. In this sense, the $\beta$-sheet content was found to be around $25 \%$ in the aggregating strains and over $40 \%$ in the non-aggregating ones (Fig. 3 and Table 3 ). These results clearly show that the secondary structures of S-layer proteins of analyzed lactobacilli play an important role in determining surface properties of these bacteria. Very interestingly, the $\beta$-sheet contents of the S-layer of the studied $L$. brevis strain (which is also non-aggregating; see Table 1 ) does not differ very much from those found for the nonaggregating $L$. kefir strains S-layer proteins, suggesting that the $\beta$-sheets play a major role in the aggregation mechanism of
Table 4

Lactobacilli S-layer DSC transition temperatures (Tm)

\begin{tabular}{llr}
\hline Bacterial strain & \multicolumn{2}{l}{ Tm of DSC transition $\left({ }^{\circ} \mathrm{C}\right)$} \\
\cline { 2 - 3 } & $1 \mathrm{st}$ & 2nd \\
\hline L. kefir CIDCA 8321 & 67.2 & 118.7 \\
L. kefir CIDCA 8348 & 69.1 & 114.5 \\
L. kefir JCM 5818 & 70.0 & 116.6 \\
L. kefir CIDCA 83113 & 68.1 & 110.4 \\
L. kefir CIDCA 8344 & 81.5 & \\
L. brevis ATCC 8287 & 57.9 & 98.2 \\
\hline
\end{tabular}

lactobacilli. On the other hand, the results also indicate that the $\alpha$ helix contents of S-layer proteins do not seem to be particularly relevant in determining the aggregation capacities of the lactobacilli strains.

\subsection{Secondary structure changes upon S-layer proteins' phase transitions}

FTIR spectroscopy also provides a valuable method for studying phase transitions in proteins, because the frequencies, intensities and bandwidths of the characteristic bands in a protein IR spectrum are very sensitive to protein conformational changes [24]. Therefore, a thermal analysis of the lyophilized S-layer proteins, within the range $30-130{ }^{\circ} \mathrm{C}$, has been carried out by FTIR, complemented by DSC measurements.

DSC analysis showed two transitions for the studied lyophilized S-layer proteins within the $30-130{ }^{\circ} \mathrm{C}$ range, with maxima located at 58 and $98{ }^{\circ} \mathrm{C}$ for $L$. brevis ATCC 8287 and in the $67-70{ }^{\circ} \mathrm{C}$ and
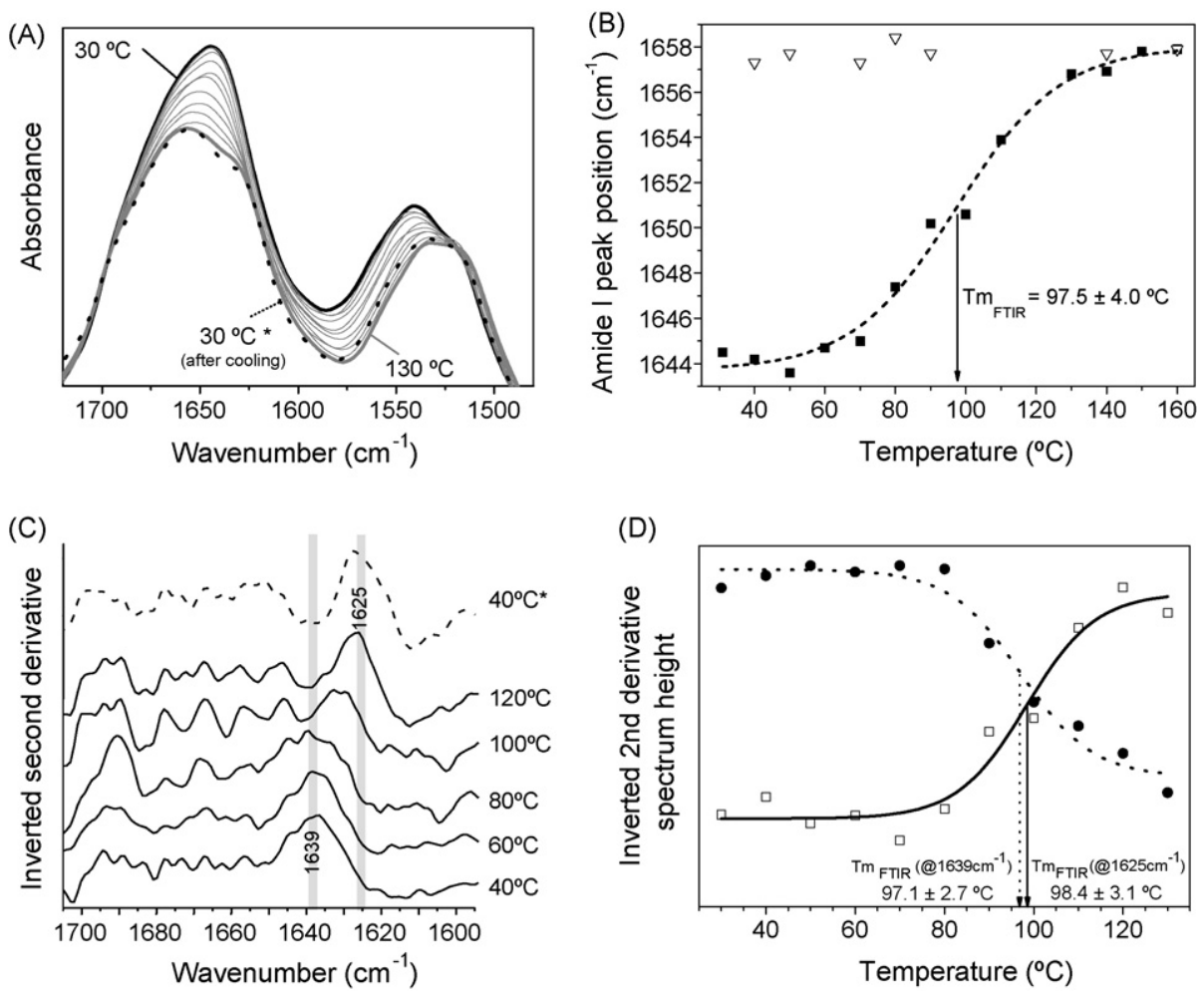

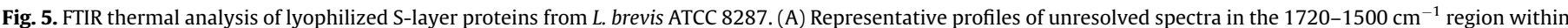

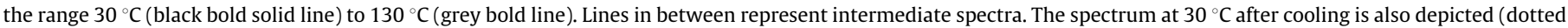

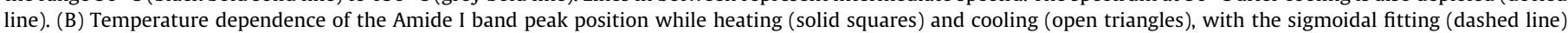

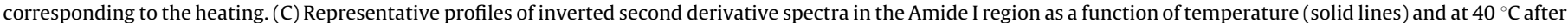

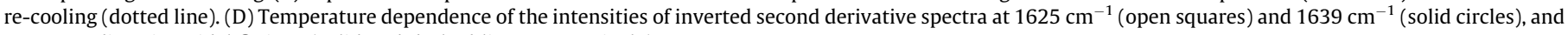
corresponding sigmoidal fittings (solid and dashed lines, respectively). 
$110-119{ }^{\circ} \mathrm{C}$ range for $L$. kefir strains, with the only exception of CIDCA 8344 strain, which seems to exhibit a single transition at ca. $81^{\circ} \mathrm{C}$, slightly higher than that of the first transition for the other Slayer proteins of the studied L. kefir strains (Fig. 4, Table 4). These transformations were found to be not reversible, as no transitions were observed during cooling and subsequent re-heating of the samples.
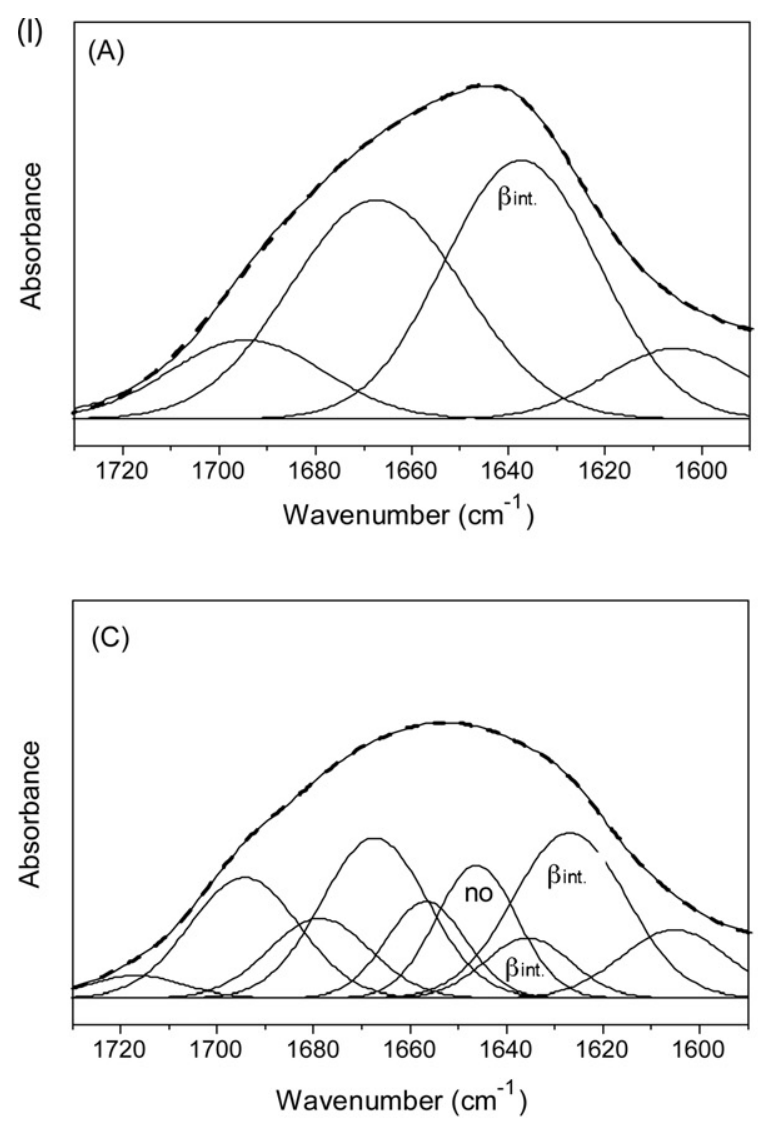

FTIR spectra reveal that, upon heating, perturbations in the secondary structure of S-layer proteins were induced. In fact, the Amide I maximum shifts to higher frequencies and the Amide II maximum (1500-1550 $\mathrm{cm}^{-1}$ range) shifts to lower frequencies as temperature increases. In addition, some other specific secondary structure related modes in second derivative spectra are also affected upon increasing the temperature [12; see also Figure 5 of
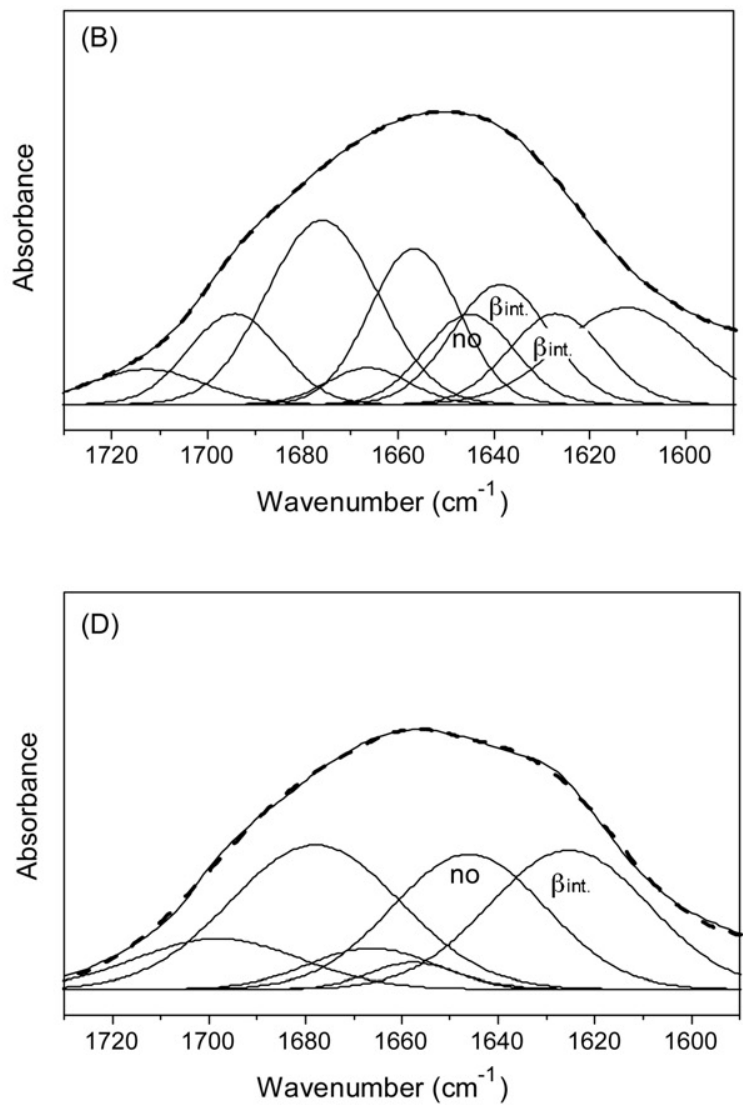

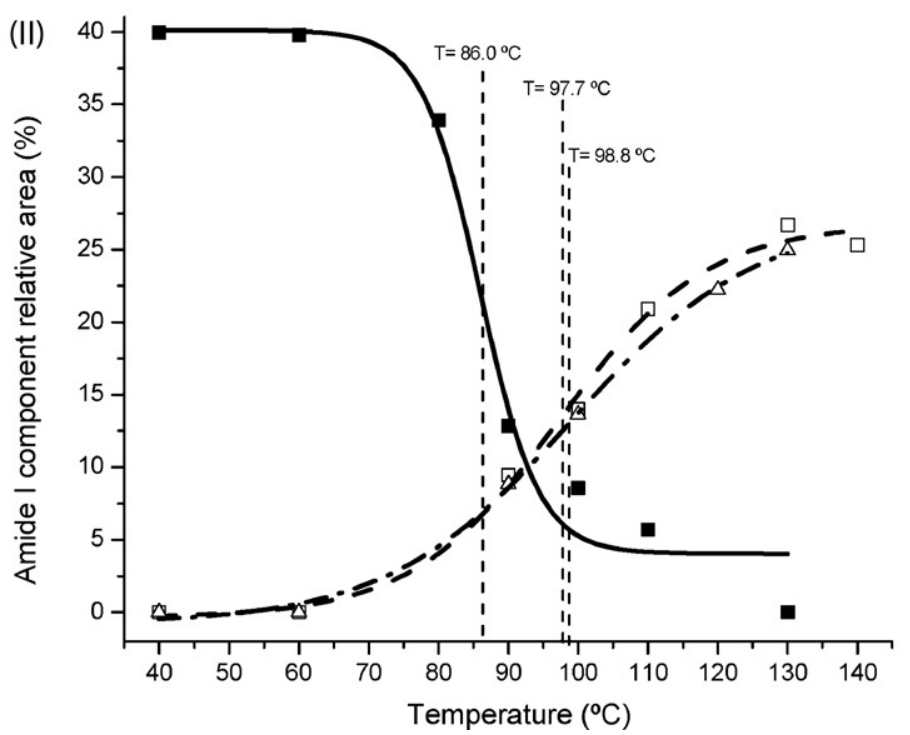

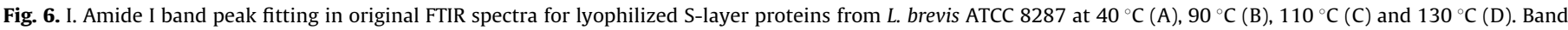

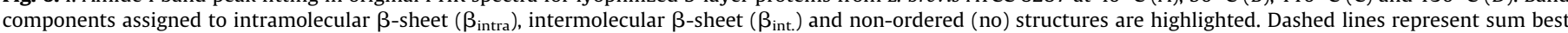

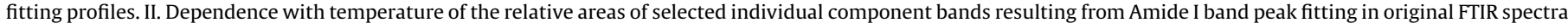

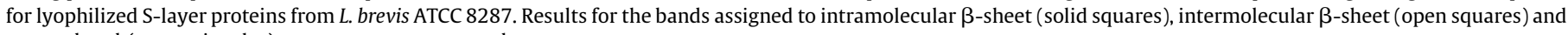
non-ordered (open triangles) structures are presented. 
this work]. In agreement with the DSC results, the observed spectroscopic changes were found to be irreversible. The phase transition temperatures $\left(\mathrm{Tm}_{\mathrm{FTIR}}\right)$ were estimated from the sigmoidal fitting midpoint of the dependence with temperature of Amide I maximum in the original spectra or of specific structure related modes in second derivative spectra [12].

The thermal analysis for $L$. brevis ATCC 8287 by FTIR is depicted in Fig. 5. As temperature increases, the Amide I maximum moves towards higher wave numbers and the Amide II maximum moves towards lower wave numbers, both decreasing in intensity (Fig. 5A). The plot of the Amide I frequency vs. temperature (Fig. 5B) showed a $\mathrm{Tm}_{\mathrm{FTIR}}$ of $97.5 \pm 4.0^{\circ} \mathrm{C}$. Inverted second derivative spectra (Fig. 5C) shows an increase in the intensity of the component band centered at $1625 \mathrm{~cm}^{-1}$ (assigned to antiparallel intermolecular $\beta$-sheets) and a decrease of the intensity of the $1639 \mathrm{~cm}^{-1}$ component (assigned to intramolecular $\beta$-sheets) with the increase of the temperature. The $\mathrm{Tm}_{\mathrm{FTIR}}$ obtained from plotting the intensity of these component bands $v$ s. temperature (Fig. 5D) were $98.4 \pm 3.1{ }^{\circ} \mathrm{C}\left(1625 \mathrm{~cm}^{-1}\right)$ and $97.1 \pm 2.7^{\circ} \mathrm{C}\left(1639 \mathrm{~cm}^{-1}\right)$. These $\mathrm{Tm}_{\mathrm{FTIR}}$ are similar to the $\mathrm{Tm}$ of the second transition measured in the DSC experiments $\left(98.2{ }^{\circ} \mathrm{C}\right)$ (Fig. 4 and Table 4). At the temperature of the first phase transition observed in the DSC experiments $\left(\mathrm{ca} .58^{\circ} \mathrm{C}\right)$, no relevant spectral changes were observed, indicating that for this strain the first phase transition shall only imply minor changes in the protein secondary structure.

Similar conclusions can be extracted performing the analysis by means of the band-deconvolution technique. Fig. 6I depicts the results of band deconvolution in the Amide I spectral region for the S-layer protein of $L$. brevis ATCC 8287 at four different temperatures. The changes in the relative areas of each component of the Amide I band upon temperature increase are plotted in Fig. 6II. The data clearly reveal the above noticed increase in the unordered and $\beta$-sheet intermolecular structures at expenses of $\beta$-sheet intramolecular structures, which dominate the secondary structure at the lowest temperatures. These trends are in agreement with the expected protein association and disordering upon denaturation. In agreement with the DSC results (Fig. 4) and also with the shift of the whole Amide I band maximum upon increasing the temperature (Fig. 5B), the sigmoidal fitting midpoint of the dependence of the intensity of the relevant component bands resulting from band deconvolution analysis with temperature occurs at $\sim 98^{\circ} \mathrm{C}$.

A similar analysis was performed for the S-layer proteins of the studied L. kefir strains. Fig. 7 summarizes the results obtained for the aggregative strain L. kefir CIDCA 8321. As discussed above in this paper, contrarily to the $L$. brevis S-layer protein, in which $\alpha$ helix structures are absent, $L$. kefir S-layer proteins have a non-

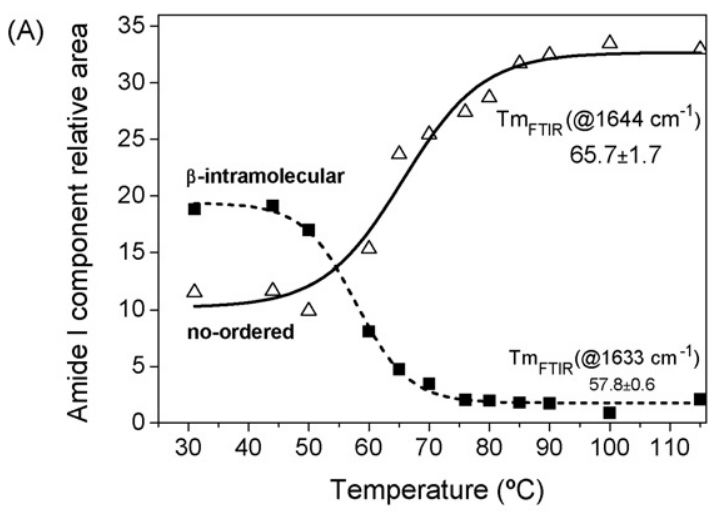

neglectable percentage of $\alpha$-helix structures (for $L$. kefir CIDCA 8321: $13.6 \%$ of the whole secondary structure; see Table 3 ). The analysis of the temperature dependence exhibited by the band at $1654 \mathrm{~cm}^{-1}$, corresponding to $\alpha$-helix structures, indicates a transition temperature (as measured by the temperature value corresponding to the midpoint of the sigmoidal fit) of $c a .60^{\circ} \mathrm{C}$, then showing a decrease of the $\alpha$-helix contents after the first phase transition observed in the DSC experiments. At the same time, a decrease in the contents of $\beta$-sheet intramolecular structures was also observed, whereas an increase of $\beta$-sheet intermolecular structures and non-ordered structures could also be noticed. Very interestingly, no significant changes could be observed at the temperature corresponding to the second phase transition peak detected by DSC (around $119{ }^{\circ} \mathrm{C}$; see Table 4). In summary, the FTIR analysis indicates that for this S-layer protein the major secondary structure rearrangements leading to denaturation took place at the first phase transition.

It is interesting to note that compared to the S-layer protein of $L$. brevis ATCC 8287 (no $\alpha$-helix contents), the protein of the $L$ kefir CIDCA 8321 strain shows a considerably smaller increase of intermolecular $\beta$-sheet structures upon aggregation. Indeed, it has been found to be common that proteins with larger content of $\alpha$ helix structure are less prone to the formation of $\beta$-sheet type aggregates $[12,49]$. This has been considered to be a consequence of the larger dipole moment of $\alpha$-helices when compared to intramolecular $\beta$-sheet structures, thus, making intermolecular interactions through the $\alpha$-helix energetically more favorable.

The analysis of the dependence of the FTIR spectra with temperature performed on the S-layer extracted from the nonaggregative strain L. kefir CIDCA 8344 (Fig. 8) reveals that the major structural changes for this protein (aggregation with increase in the intermolecular $\beta$-sheet contents and disordering, at expenses of both $\alpha$-helix and intramolecular $\beta$-sheets structures) take place at ca. $70{ }^{\circ} \mathrm{C}$ (Fig. 8), i.e., close to its phase transition detected by DSC. This phase transition is clearly correlatable with the first phase transition observed for all the other L. kefir S-layer proteins investigated. Then, at least regarding this point, the S-layer proteins show a similar behavior regardless they are aggregative or non-aggregative. The slightly higher phase transition temperature observed for the non-aggregative S-layer proteins when compared with the aggregative ones might be related with its increased contents of $\beta$-sheet structures (see Table 3 ), in consonance with previous results published for other proteins $[50,51]$. This is also in agreement with the fact that it is precisely the $L$. brevis S-layer protein, where $\alpha$-helix structures are not present and $\beta$-sheet structures reach a maximum of importance among the investi-
(B)

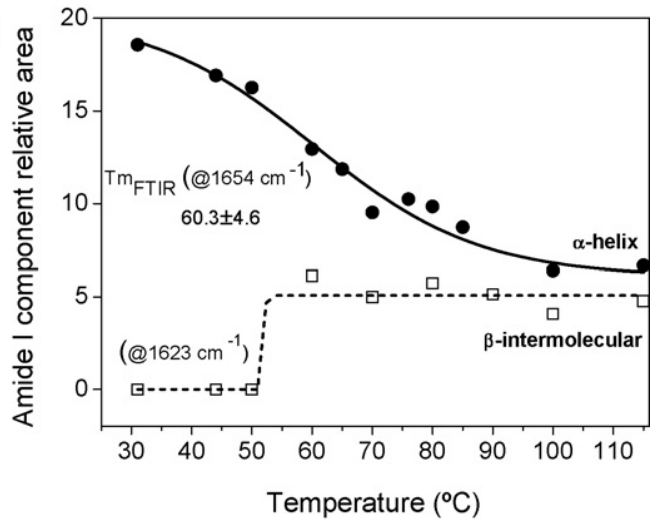

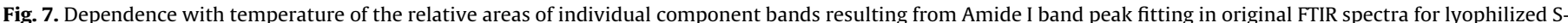

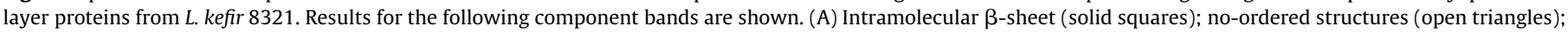
(B): $\alpha$-helix (solid circles); intermolecular $\beta$-sheet (open squares). 

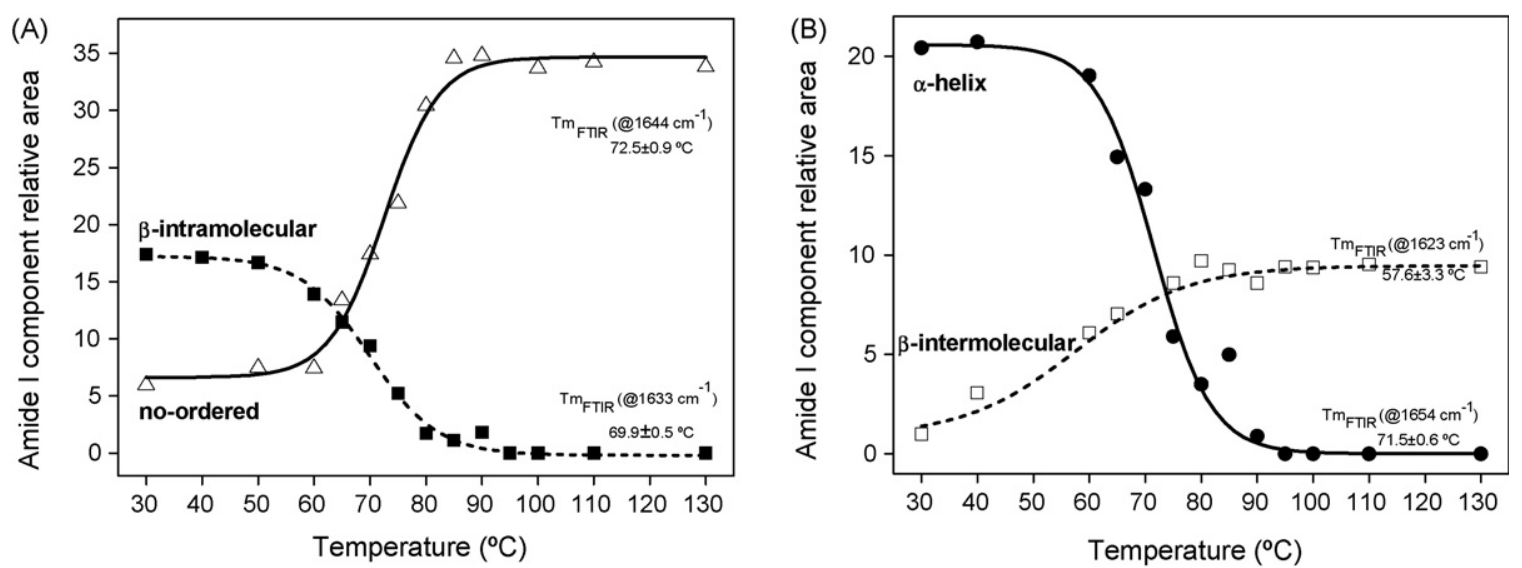

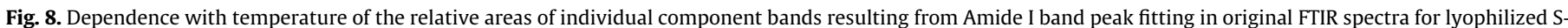

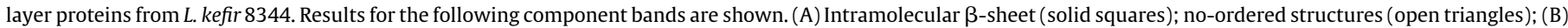
$\alpha$-helix (solid circles); intermolecular $\beta$-sheet (open squares).

gated proteins, the one that was found to be more resistant to the thermal treatment.

\section{Conclusion}

FTIR spectroscopy was used for the study of the secondary structure of S-layer proteins from lactobacilli. A high content of $\beta$ sheet structure (50.2\%) with no $\alpha$-helix structures was found for $L$. brevis S-layer proteins, in good agreement with predictions based on its primary structure ( $\beta$-sheet ranging from 44.4 [45] to $55.6 \%$ [44]; $\alpha$-helix $0 \%$ ). Characterization of the secondary structures of the $L$. kefir S-layer proteins was undertaken as for those of the $L$. brevis strain. It was found that the relative amounts of the different structural elements of the secondary structure of the investigated S-layers proteins could be correlated with the surface properties of the corresponding strains, previously determined in our laboratories [8]. A larger content of $\beta$-sheet structures was found for the S-layer proteins of the studied non-aggregating $L$. kefir strains and L. brevis.

Thermal analysis of the proteins carried out by FTIR spectroscopy allowed the assignment of most significant changes in their secondary structures at the phase transition temperatures measured by DSC. The FTIR spectra obtained as a function of temperature clearly revealed an increase in the unordered and $\beta$ sheet intermolecular structures at expenses of $\beta$-sheet intramolecular structures (which dominate the secondary structure at the lowest temperatures) with temperature increase. These trends are in agreement with the expected protein association and disordering upon denaturation. In addition, it was also found that, regardless they correspond to aggregative or non-aggregative lactobacilli strains, all investigated S-layer proteins experiment major secondary structure reorganization at a temperature that can be roughly correlated with the amount of $\beta$-sheet they contain. Thus, the slightly higher phase transition temperature observed for the non-aggregative S-layer proteins when compared with the aggregative ones might be related with its increased contents of $\beta$ sheet structures, the $L$. brevis S-layer protein, where $\alpha$-helix structures are not present and $\beta$-sheet structures reach a maximum of importance among the investigated proteins, corresponding to the thermally more resistant protein.

\section{Acknowledgements}

This work was supported by FCT (Projects POCTI/QUI/59019/ 2004 and POCTI/QUI/58937/2004), Instituto de Investigação Inter- disciplinar of the University of Coimbra (Project III/BIO/40/2005), Agencia Nacional de Promoción Científica y Tecnológica (Project PICT/2006/68), Dupont-Conicet Prize 2005 and MinCyT-FCT (Project: "Chemical and functional characterization of S-layer proteins from Lactobacillus kefir"). AGZ is member of the research career CONICET (National Research Council, Argentina). GDA is member of the research career CIC-PBA (Commission for Scientific Research, Buenos Aires). P.M. acknowledges the Program Alban, the European Union Program of High Level Scholarships for Latin America, scholarship no. E06D102040AR and CONICET. Cyted Program (Science and Technology for the Development) 108RT0362.

\section{References}

[1] G. Vidgren, I. Palva, R. Pakkanen, K. Lounatmaa, A. Palva, J. Bacteriol. 174 (1992) 7419.

[2] A. Zourari, E.M. Anifantakis, Le Lait. 68 (1988) 373.

[3] V.M. Marshall, J. Soc. Dairy Technol. 46 (1993) 49.

[4] G.L. Garrote, A.G. Abraham, G.L. De Antoni, Lebensm -Wiss. u. -Technol. 30 (1997) 77.

[5] G.L. Garrote, A.G. Abraham, G.L. De Antoni, J. Dairy Res. 68 (2001) 639.

[6] U.B. Sleytr, T.J. Beveridge, Trends Microbiol. 7 (1999) 253.

[7] M. Sára, U.B. Sleytr, J. Bacteriol. 182 (2000) 859.

[8] G.L. Garrote, L. Delfederico, R. Bibiloni, A.G. Abraham, P.F. Pérez, L. Semorile, G.L. De Antoni, J. Dairy Res. 71 (2004) 222.

[9] M. Kahala, K. Savijoki, A. Palva, J. Bacteriol. 179 (1997) 284.

[10] A. Dong, P. Huang, W.S. Caughey, Biochemistry 29 (1990) 3303.

[11] N. Takeda, M. Kato, Y. Taniguchi, Biochemistry 34 (1995) 5980.

[12] S. Matheus, W. Friess, H.Chr. Mahler, Pharm. Res. 23 (2006) 1350.

[13] J. Xie, C. Riley, M. Kumar, K. Chittur, Biomaterials 23 (2002) 3609.

[14] K. Chittur, Biomaterials 19 (1998) 357.

[15] S.-S. Cheng, K. Chittur, Ch. Krishnan, L.A. Sukenik, K. Culp, J. Lewandowska, Colloid Interf. Sci. 162 (1994) 135.

[16] W.-J. Yang, P.R. Griffiths, D. Michael Byler, H. Susi, Appl. Spectrosc. 39 (1985) 282

[17] D.M. Byler, H. Susi, Biopolymers 25 (1986) 469.

[18] H. Susi, D.M. Byler, Appl. Spectrosc. 42 (1988) 819.

[19] B.C. Smith, Fundamentals of Fourier Transform Infrared Spectroscopy, CRC Press, Boca Raton, FL, 1996.

[20] J.K. Kauppinen, D.J. Moffatt, H.H. Mantsch, D.G. Cameron, Appl. Spectrosc. 35 (1986) 271.

[21] D.G. Cameron, D.J. Moffatt, J. Test. Eval. JTEVA 12 (1984) 78.

[22] H.R. Costantino, K.G. Carrasquillo, R.A. Cordero, M. Mumenthaler, C.C. Hsu, K. Griebenow, J. Pharm. Sci. 87 (1998) 1412.

[23] H. Torii, M. Tasumi, in: H. Mantsch, D. Chapman (Eds.), Infrared Spectroscopy of Biomolecules, Wiley-Liss Inc., MA, USA, 1996, , pp. 1-18, Chapter 1.

[24] K. Murayama, M. Tomida, Biochemistry 43 (2004) 11526.

[25] A. Lupas, H. Engelhardt, J. Peters, U. Santarius, S. Volker, W. Baumeister, J. Bacteriol. 176 (1994) 1224

[26] K. Fahmy, M. Merroun, K. Pollmann, J. Raff, O. Savchuk, Biophys. J. 91 (2006) 996

[27] J.C. de Man, M. Rogosa, M.E. Sharpe, J. Appl. Bacteriol. 23 (1960) 130.

[28] G.L. Garrote, M.A. Serradell, A.G. Abraham, M.C. Anon, C.A. Fossati, G.L. De Antoni, Food Agric. Immunol. 16 (2005) 221-233.

[29] M. van de Weert, P.I. Haris, W.E. Hennink, D.J. Crommelin, Anal. Biochem. 297 (2001) 160. 
[30] P.O. Souillac, C.R. Middaugh, J.H. Rytting, Int. J. Pharm. 235 (2002) 207.

31] R. Sokal, C. Michener, Univ. Kansas Sci. Bull. 38 (1958) 1409.

[32] A. Gómez-Zavaglia, A. Abraham, S. Giorgieri, G.L. De Antoni, J Dairy Sci. 82 (1999) 870.

[33] P.J. De Urraza, A. Gómez-Zavaglia, M.E. Lozano, V. Romanowski, G.L. De Antoni, J Dairy Res. 67 (2000) 381.

[34] A. Gómez-Zavaglia, G. Kociubinski, P. Pérez, G.L. De Antoni, J. Food Protect. 61 (1998) 865.

[35] R. Sabbah, A. Xu-Lou, J.S. Chickos, M.L.P. Leitão, M.V. Roux, L.A. Torres, Thermochim. Acta 331 (1999) 93

[36] P.F. Pérez, J. Minnaard, E.A. Disalvo, G.L. De Antoni, Appl. Environ. Microbiol. 64 (1998) 21.

[37] B. Kos, J. Šuškovic, S. Vukovic, M. Šimpraga, J. Frece, S. Matošic, J. Appl. Microbiol. 94 (2003) 981.

[38] M.A. Golowczyc, P. Mobili, G.L. Garrote, A.G. Abraham, G.L. De Antoni, Int. J. Food Microbiol. 118 (2007) 264.

[39] B. Dziuba, A. Babuchowski, D. Nałęcz, M. Niklewicz, Int. Dairy J. 17 (2007) 183.

[40] A. Bosch, M.A. Golowczyc, A.G. Abraham, G.L. Garrote, G.L. De Antoni, O. Yantorno, Int. J. Food Microbiol. 111 (2006) 280.
[41] N. Thomas, R. Goodacre, E.M. Timmins, M. Gaudoin, R. Fleming, Hum. Reprod. 15 (2000) 1667

[42] A. Salman, J. Ramesh, V. Erukhimovitch, M. Talyshinsky, Sh. Mordechai, M. Huleihel, J. Biochem. Biophys. Meth. 55 (2003) 141.

[43] P. Lasch, W. Haensch, E.N. Lewis, L.H. Kidder, D. Naumann, Appl. Spectrosc. 56 (2002) 1 .

[44] B. Rost, C. Sander, J. Mol. Biol. 232 (1993) 584 (http://www.predictprotein.org).

[45] V. Brendel, P. Bucher, I.R. Nourbakhsh, B.E. Blaisdell, S. Karlin, Proc. Natl. Acad. Sci. U.S.A. 89 (1992) 2002

[46] S.J. Sillanpää, B. Martínez, J. Antikainen, T. Toba, N. Kalkkinen, S. Tankka, K. Lounatmaa, J. Keränen, M. Höök, B. Westerlund-Wikström, P.H. Pouwels, T.K. Korhonen, J. Bacteriol. 182 (2000) 6440.

[47] M. Ventura, I. Jankovic, D.C. Walker, R.D. Pridmore, R. Zink, Appl. Environ Microbiol. 68 (2002) 6172.

[48] S. Åvall-Jääskeläinen, A. Palva, FEMS Microbiol. Rev. 29 (2005) 511.

[49] E. Querol, J.A. Perez-Pons, A. Mozo-Villarias, Protein Eng. 9 (1996) 265.

[50] H. Zhang, Y. Ishikawa, Y. Yamamoto, R. Carpentier, FEBS Lett. 426 (1998) 347.

[51] E.N. Baker, R.E. Hubbard, Prog. Biophys. Mol. Biol. 44 (1984) 97. 